\title{
Gender differences in suicidal behaviour in patients with first episode psychosis
}

\author{
Gudrun Austad
}

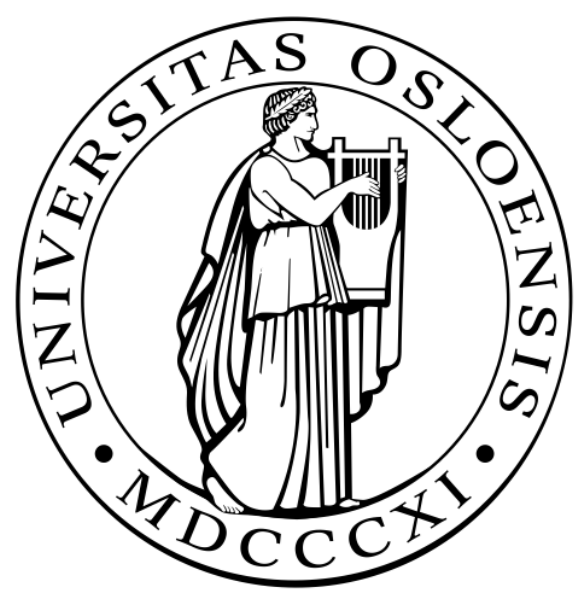

Masteroppgave (artikkel) ved Nasjonalt senter for

selvmordsforskning og -forebygging / Institutt for klinisk medisin.

UNIVERSITETET I OSLO

Mai, 2011 


\section{Abstract}

Introduction: In the early phases of psychosis there is a high risk of suicide and suicidal behaviour. Previous research on psychotic disorders has shown contradicting gender patterns in suicidal behaviour. This study aims to describe the prevalence of suicidal behaviour and analyze this according to gender and risk factors for suicidal behaviour in a group of consecutively included patients with first episode nonaffective psychosis (FEP).

Method: Two hundred and nineteen patients from an area with a program for early detection (ED) of psychosis were examined, and data from baseline were used for analyzes. Social, demographical and clinical variables were analyzed and compared between genders. Suicidal behaviour was rated at baseline and retrospectively reported as levels of suicidality (suicidal ideations, suicidal plans or suicide attempts) for two time periods; last month prior to study entry and in lifetime.

Results: Suicidal behaviour in lifetime was present in $66.1 \%$ of the patients, while $50.9 \%$ reported suicidal behaviour during last month. Females had significantly higher prevalence of suicidal behaviour than males at both time intervals. Suicidal behaviour was associated with depressive symptoms and female gender and remained significant when included in a multivariate model.

Conclusion: Suicidal behaviour was highly prevalent in this sample of FEP patients with higher levels of suicidality in females than in males. Depressive symptoms and female gender were associated with suicidal behaviour.

Key words: psychotic disorders, schizophrenia, suicide, suicide attempted, suicidal behaviour, gender differences 


\section{Introduction}

\subsection{Prevalence of suicide and suicidal behaviour in the general population}

Suicide is a major health problem, with a global mortality rate of 16 per 100000 , almost one million suicides per year (WHO 2011). Suicide accounts for $1.4 \%$ of all deaths globally (Murray and Lopez 1996; WHO 2004). Suicidal behaviour can be seen as a continuum from suicidal ideations via suicidal plans to suicide attempts, with an elevated risk of transition from ideation to plan and from plan to attempt (Kessler, Borges et al. 1999; Nock, Borges et al. 2008). Suicidal ideations and suicide attempts are risk factors for later suicide attempts and completed suicide (Beghi and Rosenbaum 2010; Cavanagh, Carson et al. 2003; Bernal, Haro et al. 2007). A WHO study reported a lifetime prevalence of suicidal ideations from $2-25 \%$, suicidal plans from $1-16 \%$ and suicide attempts from $0.4-4 \%$ in the general population of 8 European countries (Bertolote, Fleischmann et al. 2005). In a large study with respondents from 17 countries, the cross-national lifetime prevalence of suicidal ideations, -plans and -attempts were $9 \%, 3 \%$ and $3 \%$ respectively (Nock, Borges et al. 2008). The number of suicide attempts is estimated to be 10 - 20 times the suicide rate (Welch 2001; Casey, Dunn et al. 2008).

\subsection{Gender differences in suicide and suicidal behaviour in the general population}

Suicide and suicidal behaviour are related to gender. In most countries males more often commit suicide, while suicidal ideations and non-fatal suicidal behaviour are more prevalent in females (Canetto 1997; Weissman, Bland et al. 1999; Canetto 2008; Nock, Borges et al. 2008). The fact that suicidal behaviour is more prevalent in females while the majority of suicides are completed by males, has been labelled the 
"gender paradox of suicide" (Canetto and Sakinofsky 1998). The male: female suicide rate varies across countries with an average of 3.5:1 (WHO 2011b). In the "WHO/EURO Study on Parasuicide" the average suicide attempt rates for females were 1.5 times higher than for males (Kerkhof 2000).

\subsection{Prevalence of suicide and suicidal behaviour in psychosis}

Patients with psychotic disorders represent a high risk group concerning suicidal behaviour and completed suicide (Radomsky, Haas et al. 1999; Melle, Johannesen et al. 2006; Tidemalm, Langstrom et al. 2008). As in the general population, suicidal ideations and previous suicide attempts are risk factors for later suicide attempts and completed suicide in patients with psychotic disorders (Tarrier, Barrowclough et al. 2004; Hawton, Sutton et al. 2005). The lifetime risk of suicide in schizophrenia is 4 $5 \%$, with the highest risk in the early course of the disorder (Inskip, Harris et al. 1998; Palmer, Pankratz et al. 2005). The prevalence of suicidal ideations and suicide attempts is also high in patients with psychotic disorders. Suicidal ideations have been detected in 25 - $40 \%$ of patients with first episode psychosis (FEP) at baseline (Tarrier, Barrowclough et al. 2004; Hocaoglu and Babuc 2009; Melle, Johannessen et al. 2010). Up to $50 \%$ of patients with psychotic disorders have a lifetime history of suicide attempts (Radomsky, Haas et al. 1999; Tarrier, Barrowclough et al. 2004). In patients with FEP 5 - $28 \%$ report suicide attempt prior to start of treatment (Cohen, Lavelle et al. 1994; Addington, Williams et al. 2004; Cougnard, Kalmi et al. 2004; Clarke, Whitty et al. 2006; Melle, Johannesen et al. 2006; Harvey, Dean et al. 2008; Robinson, Cotton et al. 2009). The prevalence of completed suicide, suicidal ideations and suicide attempts are consistently higher in patients with psychotic disorders than in the general population (Tarrier, Barrowclough et al. 2004; Hocaoglu and Babuc 2009; Melle, Johannessen et al. 2010). 


\subsection{Gender differences in suicide and suicidal behaviour in psychosis}

The gender pattern for suicide and suicidal behaviour in psychotic disorders differs somewhat from the general population. Regarding completed suicide, most studies on schizophrenia demonstrates the same gender differences as seen in the general population; more males than females die from suicide (Heila, Isometsa et al. 1997; Hiroeh, Appleby et al. 2001; Lester 2006; Pompili, Amador et al. 2007). A few studies report no gender differences in completed suicide in patients with schizophrenia (Goldstein, Santangelo et al. 1993; Carlborg, Jokinen et al. 2008; Reutfors, Brandt et al. 2009). It has been stated that gender differences in completed suicide is less marked for patients with psychotic disorders than seen in the general population, with an odds ratio for suicide in men of 1.57 (Hawton, Sutton et al. 2005).

Research on gender differences in suicidal ideations in FEP is limited. One study of FEP patients reported no gender differences in suicidal ideations (Thorup, Petersen et al. 2007), while another study reported higher prevalence of suicidal ideation in females (Barrett, Sundet et al. 2010). Gender differences in suicide attempts in FEP have been studied more extensively than gender differences in suicidal ideations; the results are however, ambiguous. The gender pattern in the general population, that more females make suicide attempts, is reproduced in a number of FEP studies (Nordentoft, Jeppesen et al. 2002; Bertelsen, Jeppesen et al. 2007; Cotton, Lambert et al. 2009; Barrett, Sundet et al. 2010; Melle, Johannessen et al. 2010). Contrary to these findings, several studies found no differences in suicide attempts related to gender (Haw, Hawton et al. 2005; Clarke, Whitty et al. 2006; Foley, Jackson et al. 2008; Robinson, Cotton et al. 2009). One FEP study found higher prevalence of suicide attempts in males (Harvey, Dean et al. 2008), but this seems to be an exception. Altogether, research on gender differences in suicide and suicidal behaviour in FEP gives a contradicting pattern. 


\subsection{The TIPS study}

The TIPS (early Treatment and Intervention in PSychosis) study was a prospective longitudinal study of patients with FEP from four Scandinavian health care sectors with equivalent treatment programmes and a parallel control design. Two of the sectors had an extensive early detection and intervention programme (ED) and two did not (No - ED) (Johannessen, Joa et al. 2011; Melle, Larsen et al. 2004). The study explored the clinical use of an ED program in reducing the duration of untreated psychosis (DUP) in a population based catchment area in the time period January 1, 1997 to December 31, 2000. The DUP was significantly shorter for the patients coming from the ED areas, compared to patients from the No-ED areas. The reduction of DUP was also associated with a better clinical status at baseline (Melle, Larsen et al. 2004) and better short- (Larsen, Melle et al. 2006; Melle, Larsen et al. 2008) and long-term outcome (Larsen, Melle et al. 2010). The present study is conducted in the former ED area of the TIPS study in a later time period: from January 1, 2002 to March 10, 2010.

\subsection{Study aim}

The aim of this study is to describe the prevalence of suicidal behaviour i.e. suicidal ideations, suicidal plans and suicide attempts, and analyze this according to gender and risk factors for suicidal behaviour in a group of consecutively included patients with first episode non-affective psychosis (FEP). 


\section{Material and methods}

\subsection{Participants}

The inclusion criteria were as follows: Living in the catchment area (The Southern region of Rogaland County); age between 15 - 65 years; meeting the DSM-IV criteria (APA 2005) for FEP; i.e. schizophrenia, schizophreniform disorder, schizoaffective disorder (core schizophrenia spectrum disorders) or brief psychotic episode, delusional disorder, affective psychosis with mood-incongruent delusions, psychotic disorder not otherwise specified or drug induced psychosis; being actively psychotic, as measured by a Positive and Negative Syndrome Scale (PANSS) score (Kay, Fiszbein et al. 1987) of four or more on at least one of the following PANSS items: P1 (delusions), P3 (hallucinations), P5 (grandiose thinking), P6 (suspiciousness) and A9 (unusual thought content); not previously receiving adequate treatment for psychosis (defined as antipsychotic medication of 3.5 haloperidol equivalents for 12 weeks or until remission of the psychotic symptoms); no neurological or endocrine disorders with relationship to the psychosis; no contraindications to antipsychotic medication; understands/speaks one of the Scandinavian languages; intelligence quotient over 70 (WAIS); willing and able to give informed consent.

This study used the baseline characteristics from the TIPS II and III sample, a population based cohort of consecutive FEP patients recruited over one time period: January 1, 2002 to March 10, 2010 (TIPS II and III cohorts), to address the questions outlined above. The sample is recruited from one geographical area (The southern part of Rogaland County, Norway), with 380000 inhabitants living mainly in urban and suburban areas. All individuals identified as potential cases of FEP seeking help from the specialized psychiatric services were screened by the study assessment team (32/100 000 per year). A total of 787 potential first episode cases were screened during the study inclusion period. Of these, 355 were eliminated because the criterion for a diagnosis of FEP was not met. Of the remaining 432 FEP cases 67 patients did 
not meet study inclusion criteria. From the sample of 365 that were study appropriate $146(40 \%)$ individuals refused to participate. Thus, a total of 219 patients with FEP signed the informed consent form and were included in the study. All FEP patients were assessed by trained personnel within a week of first contact, assigned without delay to treatment consisting of standardized antipsychotic medication protocol, multi-family work and supportive psychotherapy (Joa, Johannessen et al. 2009).

The study was approved by the Regional Ethical Committee (REK Vest 015.03). Written informed consent was obtained from all study participants. For patients younger than 18 years of age, written informed consent was also given by their parents or legal guardians.

\subsection{Measures}

The Structured Clinical Interview for the DSM-IV Axis I Disorders (SCID) (First M 1995) was used for diagnostic purposes. Symptom levels were measured by mean scores on the PANSS (Kay, Fiszbein et al. 1987) divided into Positive, Negative, Excitement, Cognitive and Depressive components, based on a five-factor PANSS component analysis (Bentsen, Munkvold et al. 1996). Function level was measured by the Global Assessment of Functioning Scale (GAF) (APA 2005), and the scores were split into symptom (GAFs) and function (GAFf) sub scores. Abuse of alcohol and other drugs was measured by the Drake Scale, where none (1), mild (2), moderate (3), severe (4) and extremely severe (5) are used as measures (Drake, Osher et al. 1990). Alcohol abuse had a cut-off score of 3 , while drug abuse had cut-off score 2. DUP was defined as the time from onset of psychosis until the start of adequate treatment (Larsen, McGlashan et al. 1996). Onset of psychosis was equated with the first appearance of positive psychotic symptoms, defined as the first week with symptoms corresponding to a PANSS score of 4 or more on positive subscale items $1,3,5$, or 6 or on general subscale item 9. All available data sources, including semi-structured personal interviews with patients and relatives, were used to ascertain the length of 
this period. Adequate treatment was defined as the start of structured treatment with antipsychotic medications, start of hospitalization or the start of out-patient clinic psychotherapy aimed at treating psychotic symptoms. Functioning before the onset of psychosis was measured by the Premorbid Adjustment Scale (PAS) (Cannon-Spoor, Potkin et al. 1982), which covers two dimensions: Social (Social accessibilityisolation and Peer relationships) and academic (School performance and School adaptation). The scoring range of each item is $0-6$, with 0 indicating the best level of functioning and 6 the worst. We used childhood level and change (difference between last premorbid score and childhood level) for each domain in the analyses (Larsen, Friis et al. 2004). Suicidal behaviour was assessed at the index diagnostic interview. The patients were asked if they had experienced suicidal thoughts (ideations), suicidal plans, or attempted suicide at the time of contact including the previous month, and earlier in their lifetime. That is; suicidal thoughts, not only thoughts of death, plans of how to commit suicide or attempt with suicidal intent, not self harm without suicidal intent. The highest reported level of suicidality for the time interval (lifetime and last month) was registered, the variable labelled: "Level of suicidality" (Melle, Johannessen et al. 2010).

\subsection{Assessment}

The assessment team consisting of clinically experienced and trained research personnel performed all evaluations. All raters were trained in the use of study instruments by rating pre-prepared case notes and videotapes before entering the study assessment team. The rating of essential variables such as diagnosis and DUP was made by consensus with experienced clinical researchers. Reliability for the PANSS scores was measured by the rating of videotaped interviews of first episode patients by all raters. Reliability for diagnosis, GAF and DUP was measured by the rating of actual case notes by blind raters with substantial clinical research experience. Baseline inter-rater reliability was fair to good for all measures mentioned above (Joa, Johannessen et al. 2008). 


\subsection{Statistical analyses}

Analyses were performed with the Statistical Package for the Social Sciences (SPSS, version 17.0, Chicago, IL, USA). Parametric analyses were used for variables with normal distribution and reported in mean values and standard deviations (SD). Nonparametric analyses were used for variables with skewed distributions and reported in median values. For continuous variables Student $t$-tests were used for comparison between groups, and Chi-square or Fischer's exact test was used for categorical variables. DUP was not normally distributed, but its natural logarithm is. All analyses that include DUP are thus non-parametric (Mann Whitney-U test) where possible. In the logistic regression, we used the natural logarithm of DUP. Level of suicidality was treated as an ordinal variable in the analyses. All tests were two-tailed and the level of statistical significance was defined as $\mathrm{p}=<0.05$.

To examine social, demographical and clinical predictors of suicidal behaviour, and to evaluate the impact of potential confounders for the relationship between suicidal behaviour and gender, a binary logistic regression analysis was performed with nonsuicidal (no suicidal ideations, -plans or -attempts)/ suicidal (suicidal ideations, -plans and -attempts) as dependent variable. This dichotomizing was done due to the relatively small number of patients with suicide attempts and suicide plans. Social, demographical and clinical variables that differed significantly between the genders were entered as independent variables. The final model is presented in the results section. 


\section{Results}

Fifty-eight percent of the patients were males. The whole group had a mean age (Mean $[\mathrm{SD}]=27.0[10.3]$ ) corresponding to young adulthood. The populations mean premorbid childhood level of social - (1.1 [1.3]) and academic functioning (1.8 [1.2]) was slightly lowered. Their mean education length (11.8 [2.5]) was corresponding to half way through comprehensive school and a vast majority (82.6\%) of the patients were not married or cohabitant. The males had a significant greater change in premorbid social functioning than the females (Table 1), i.e. males had a greater decline in social functioning from childhood to the last premorbid level measured. No significant gender differences were found for age, childhood level of social- and academic functioning, change in premorbid academic functioning, length of education or marital status.

Table 1:

Social and demographic characteristics in patients with First Episode Psychosis at baseline sorted by gender

\begin{tabular}{|c|c|c|c|c|c|}
\hline Variable: & $\mathrm{n}$ & Total & $\begin{array}{l}\text { Male } \\
(\mathrm{n}=127)\end{array}$ & $\begin{array}{l}\text { Female } \\
(\mathrm{n}=92)\end{array}$ & $p$ \\
\hline Age at study entry (years) & $127 / 92$ & $27.0(10.3)$ & $28.4(9.1)$ & $24.9(11.4)$ & 0.110 \\
\hline \multicolumn{6}{|l|}{$\begin{array}{l}\text { Premorbid social } \\
\text { functioning }\end{array}$} \\
\hline Childhood level & $114 / 81$ & $1.1(1.3)$ & $1.4(1.9)$ & $1.8(2.2)$ & 0.261 \\
\hline $\begin{array}{l}\text { Change in premorbid } \\
\text { social functioning }\end{array}$ & $114 / 81$ & $0.8(1.6)$ & $1.1(1.7)$ & $0.5(1.5)$ & $0.024 *$ \\
\hline \multicolumn{6}{|l|}{$\begin{array}{l}\text { Premorbid academic } \\
\text { functioning }\end{array}$} \\
\hline Childhood level & $114 / 80$ & $1.8(1.2)$ & $3.3(1.9)$ & $2.8(1.8)$ & 0.102 \\
\hline $\begin{array}{l}\text { Change in premorbid } \\
\text { academic functioning }\end{array}$ & $100 / 74$ & $0.7(1.5)$ & $0.6(1.7)$ & $0.8(1.3)$ & 0.416 \\
\hline Length of education (years) & $122 / 90$ & $11.8(2.5)$ & $12.0(2.8)$ & $11.5(2.0)$ & 0.160 \\
\hline Married or cohabiting, $\mathrm{n}(\%)$ & $127 / 92$ & $38(17.4)$ & $26(21.0)$ & $12(13.5)$ & 0.211 \\
\hline
\end{tabular}

Note: All values are mean and standard deviation (SD), when not otherwise specified.

$*=$ Level of significance $=<0.05$ 
The majority of the sample was diagnosed with non-schizophrenia spectrum psychosis i.e. brief psychotic episode (5.9\%), delusional disorder (7.8\%), affective psychosis with mood-incongruent delusions (23.3\%), psychotic disorder not otherwise specified (18.3\%) or drug induced psychosis (5\%). The PANSS scores showed a symptom pattern of negative-, positive- and depressive symptoms. The mean GAF scores indicated serious symptoms and serious impairment in social and occupational functioning. Gender differences in clinical characteristics are presented in Table 2. Males had higher prevalence of schizophrenia spectrum disorders (core), longer DUP, higher scores on the PANSS positive- and cognitive components and lower scores on the PANSS depressive component compared to females. Drug abuse was significantly more frequent in males. There were no significant gender differences in the negative- and excitement components of the PANSS, in the GAF scores or in alcohol abuse.

Table 2.

Clinical characteristics in patients with First Episode Psychosis at baseline sorted by gender

\begin{tabular}{|c|c|c|c|c|c|}
\hline Variable: & $\begin{array}{l}\mathrm{n} \\
\text { Male/female }\end{array}$ & Total & $\begin{array}{l}\text { Male } \\
(n=127)\end{array}$ & $\begin{array}{l}\text { Female } \\
(n=92)\end{array}$ & $p$ \\
\hline \multicolumn{6}{|l|}{ Diagnoses, n (\%) } \\
\hline Schizophrenia spectrum disorder (core) & $127 / 92$ & $87(39.7)$ & $65(51.2)$ & $22(23.9)$ & $0.000 *$ \\
\hline DUP (weeks), median (min-max) & $123 / 90$ & $16(0-2080)$ & $20(0-2080)$ & $14.5(0-520)$ & $0.028 *$ \\
\hline \multicolumn{6}{|l|}{ PANSS components, mean (SD) } \\
\hline Negative & $126 / 91$ & $19.1(7.6)$ & $19.2(7.5)$ & $19.0(7.7)$ & 0.897 \\
\hline Positive & & $14.8(4.0)$ & $15.2(4.4)$ & $14.1(3.3)$ & $0.037 *$ \\
\hline Excitement & & $8.2(3.4)$ & $8.5(3.5)$ & $7.7(3.2)$ & 0.110 \\
\hline Depressive & & $12.7(3.8)$ & $12.0(3.6)$ & $13.6(3.9)$ & $0.002 *$ \\
\hline Cognitive & & $5.9(3.0)$ & $6.4(3.1)$ & $5.3(2.7)$ & $0.006^{*}$ \\
\hline GAF-F, mean (SD) & $127 / 91$ & $39.2(9.6)$ & $38.2(9.1)$ & $40.6(10.1)$ & 0.066 \\
\hline GAF-S & & $30.8(7.3)$ & $30.1(7.7)$ & $31.8(6.6)$ & 0.084 \\
\hline Alcohol abuse last 6 months n (\%) & $127 / 91$ & $24(11.0)$ & $14(11.0)$ & $10(11.0)$ & 1.000 \\
\hline Drug abuse last 6 months & & $75(34.2)$ & $52(40.9)$ & $23(26.1)$ & $0.036^{*}$ \\
\hline
\end{tabular}

$*=$ Level of significance $=<0.05$ 
We found high levels of suicidality in the patients, with $66.1 \%$ reporting suicidality (suicidal ideations, -plans or -attempts) during lifetime. For the period of last month $50.9 \%$ of the patients reported suicidality (Table 3 ). Females had significantly higher levels of suicidality than males for both time intervals. When dichotomizing the level of suicidality into non-suicidal (the absence of suicidal ideations, -plans or -attempts) and suicidal (presence of suicidal ideations, -plans and -attempts), we found significant gender differences both for lifetime and last month.

Table 3.

Level of suicidality in patients with First Episode Psychosis, lifetime and last month at baseline sorted by gender

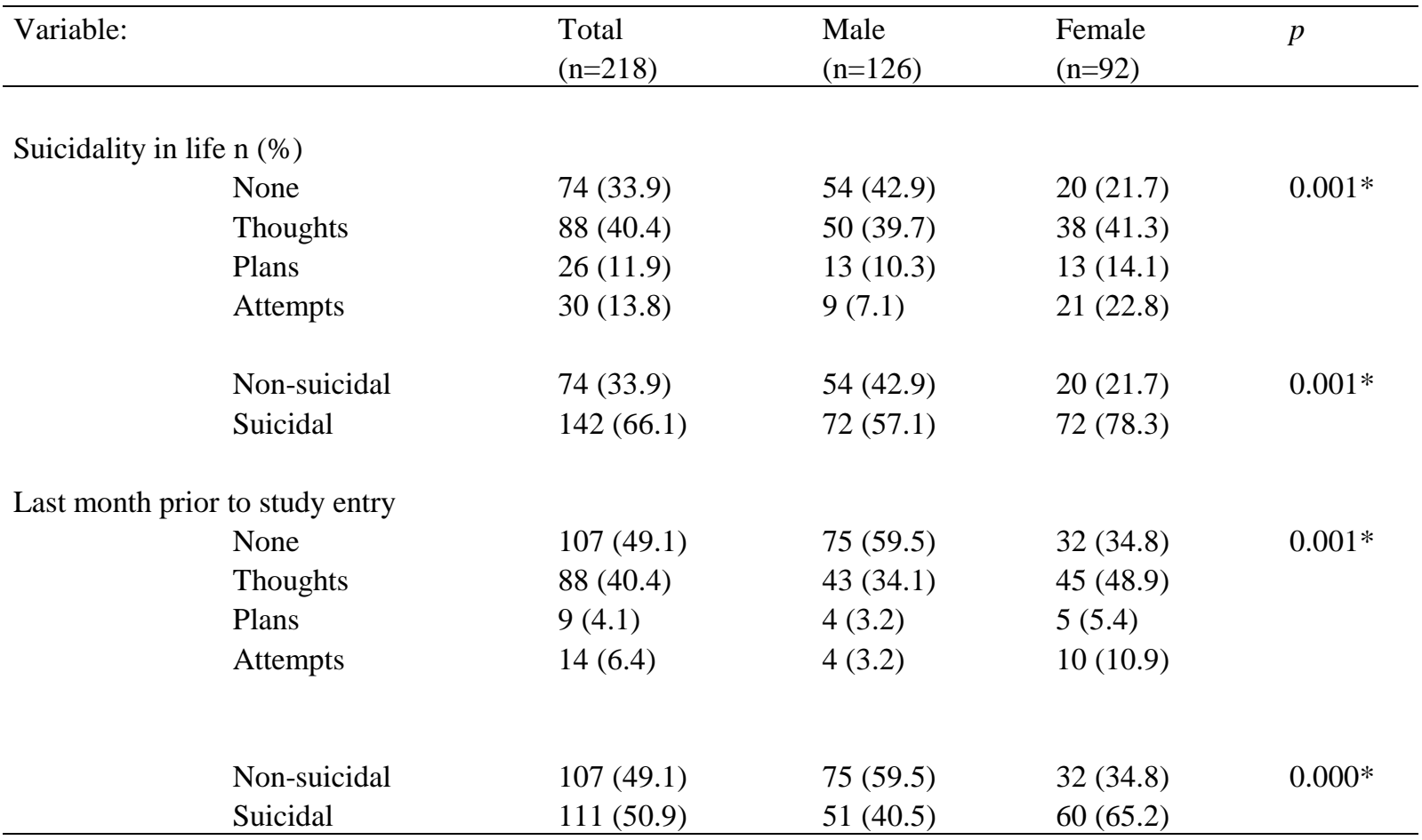

$*$ Level of significance $\mathrm{p}=<0.05$

In order to study what predicts suicidal behaviour overall, we carried out a logistic regression analysis with non-suicidal or suicidal for lifetime (Table 4) and last month (Table 5) as the dependent variable, and the variables with a significant gender difference (premorbid social dimension change score, diagnosis [schizophrenia spectrum disorder/ non-schizophrenia spectrum disorder], DUP, positive-, depressiveand cognitive components of the PANSS and drug abuse) and gender as independent 
variables. In this multivariate model depressive symptoms and female gender were associated with suicidality for both time intervals. The independent variables; premorbid social dimension change score, schizophrenia spectrum disorder (core), DUP, the positive- and cognitive component of the PANSS and drug abuse did not remain significant when included in a multivariate model. Thus, female gender and depressive symptoms significantly explains differences in suicidality even when controlling for confounders.

\section{Table 4.}

Logistic regression with suicidality (non-suicidal / suicidal) in lifetime as dependent variable for patients with First Episode Psychosis $(n=186)$

\begin{tabular}{|c|c|c|c|c|c|}
\hline Variable: & $B$ & S.E & $\mathrm{p}$ & $\operatorname{Exp}(B)$ & $95 \% \mathrm{CI}$ \\
\hline $\begin{array}{l}\text { Social function } \\
\text { change }\end{array}$ & 0.133 & 0.113 & 0.239 & 1.142 & $0.915-1.426$ \\
\hline $\begin{array}{l}\text { Schizophrenia } \\
\text { spectrum } \\
\text { disorder (core) }\end{array}$ & 0.496 & 0.371 & 0.181 & 1.643 & 0.793-3.402 \\
\hline $\log \mathrm{DUP}$ & 0.161 & 0.223 & 0.471 & 1.175 & $0.759-1.818$ \\
\hline PANSS & & & & & \\
\hline Positive component & 0.021 & 0.044 & 0.630 & 1.021 & $0.937-1.114$ \\
\hline Depressive component & 0.236 & 0.055 & 0.000 & 1.266 & $1.136-1.411 *$ \\
\hline Cognitive component & -0.086 & 0.060 & 0.147 & 0.917 & $0.816-1.031$ \\
\hline Drug misuse (yes/no) & 0.100 & 0.374 & 0.789 & 1.105 & $0.531-2.299$ \\
\hline Gender & 0.892 & 0.383 & 0.020 & 2.440 & $1.152-5.165^{*}$ \\
\hline
\end{tabular}


Table 5.

Logistic regression with suicidality (non-suicidal / suicidal) last month prior to study entry as dependent variable for patients with First Episode Psychosis $(n=187)$

\begin{tabular}{llllll}
\hline Variable & $B$ & S.E & $\mathrm{p}$ & $\operatorname{Exp}(B)$ & $95 \%$ CI \\
\hline $\begin{array}{l}\text { Social function } \\
\text { change }\end{array}$ & 0.140 & 0.104 & 0.181 & 1.150 & $0.937-1.411$ \\
\hline
\end{tabular}

Schizophrenia

spectrum

disorder (core)

$\begin{array}{llll}0.212 & 0.351 & 0.546 & 1.236\end{array}$

$0.621-2.457$

$\log \mathrm{DUP}$

$0.093 \quad 0.212 \quad 0.661$

1.097

$0.724-1.662$

\section{PANSS}

$\begin{array}{llllll}\text { Positive component } & -0.046 & 0.043 & 0.282 & 0.955 & 0.877-1.039\end{array}$

Depressive component $0.198 \quad 0.050 \quad 0.000 \quad 1.219 \quad 1.106-1.345^{*}$

\begin{tabular}{llllll} 
Cognitive component & -0.031 & 0.058 & 0.596 & 0.917 & $0.865-1.087$ \\
\hline
\end{tabular}

$\begin{array}{llllll}\text { Drug misuse (yes/no) } & -0.298 & 0.352 & 0.397 & 0.742 & 0.372-1.480\end{array}$

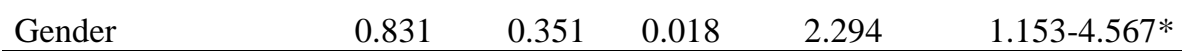

$\operatorname{logDUP}=$ duration of untreated psychosis $\log$ transformed. Model Chi Square $=35.273, \mathrm{df}=8, \mathrm{pb} .001$.

The model as a whole explained between $17.2 \%$ (Cox and Snell R2) and 22.9\% (Nagelkerke R2) of the variance and correctly indentified $67.9 \%$ of the cases. 


\section{Discussion}

The main finding of this study was a high prevalence of suicidal behaviour in FEP patients, both in lifetime and last month. We also found higher levels of suicidality in females for lifetime and last month. Depressive symptoms and female gender were associated with higher levels of suicidality.

\subsection{Prevalence of suicidal behaviour}

Overall we found a high prevalence of suicidal behaviour in the FEP patients. The lifetime prevalence rates of suicidal ideations we report are similar to other FEP results, about 40 \% (Fenton 2000; Nordentoft, Jeppesen et al. 2002; Barrett, Sundet et al. 2010). We found a lower lifetime prevalence of suicide attempts (13,8\%) than reported in comparable FEP samples, ranging from 24 - $28 \%$ (Bertelsen, Jeppesen et al. 2007; Barrett, Sundet et al. 2010; Robinson, Harris et al. 2010). It was not possible to compare the prevalence of suicidal behaviour for the period of last month with other FEP studies, apart from the TIPS study, due to differences in study designs. Compared to findings in the TIPS study, we detected higher levels of suicidality in our sample compared to the reports of Melle and colleagues from the ED area, both for lifetime and last month. The levels of suicidality found in our study was similar to the No-ED areas of the TIPS study for the period of last month, but higher than the suicidality levels in the No-ED areas for lifetime (Melle, Johannesen et al. 2006).

The high prevalence of all suicidal behaviour in our study is consistent with the well documented heightened suicide risk in the early phases of schizophrenia (Harris and Barraclough 1997; Palmer, Pankratz et al. 2005). The lower prevalence of suicide attempts in our study may be explained by the relatively short DUP in this sample compared to other FEP samples (Bertelsen, Jeppesen et al. 2007; Robinson, Cotton et al. 2009; Barrett, Sundet et al. 2010). 


\subsection{Gender differences in social, demographical and clinical characteristics}

Regarding gender differences in social, demographical an clinical characteristics, we found that males had a decline in premorbid social functioning from childhood until onset of psychosis, a higher prevalence of schizophrenia spectrum disorders (core), longer DUP, more symptoms (positive- and cognitive components of the PANSS) and more drug abuse. Females had higher scores on the depressive component of the PANSS. These findings are consistent with gender differences in other FEP studies for the variables premorbid social functioning (Bakst, Rabinowitz et al. 2010b; Preston, Orr et al. 2002; Thorup, Petersen et al. 2007), depressive symptoms (Thorup, Petersen et al. 2007; Koester, Lajer et al. 2008; Cotton, Lambert et al. 2009) and drug abuse (Thorup, Petersen et al. 2007; Koester, Lajer et al. 2008; Cotton, Lambert et al. 2009). The gender differences we found in schizophrenia spectrum disorders (core), DUP and the positive- and cognitive components of the PANSS was not found in other FEP studies (Preston, Orr et al. 2002; Thorup, Petersen et al. 2007; Koester, Lajer et al. 2008; Cotton, Lambert et al. 2009). Research on gender differences in FEP is scarce. Due to differences in study designs and assessment instruments, a full comparison of social, demographical and clinical variables in our cohort to cohorts of other FEP studies is difficult.

\subsection{Gender differences in suicidal behaviour}

Gender differences in suicidal behaviour found in our sample, with higher levels of suicidality in females, is consistent with the gender pattern of suicidal behaviour in the general population (Weissman, Bland et al. 1999; Nock, Borges et al. 2008) and in several FEP studies (Thorup, Petersen et al. 2007; Cotton, Lambert et al. 2009; Barrett, Sundet et al. 2010). It has been suggested that the severity of a clinical condition like schizophrenia, to some extent, could override the gender differences in suicidal behaviour expected from observations in the general population (Carlborg, Winnerback et al. 2010), and thus serve as an explanation for the lack of gender 
differences in suicidal behaviour found in several studies (Haw, Hawton et al. 2005). Some of the FEP studies reporting no gender differences in suicidal behaviour have a median DUP ranging from 20 to 50 weeks (Clarke, Whitty et al. 2006; Thorup, Petersen et al. 2007; Robinson, Cotton et al. 2009). This means that at baseline the patients had been ill with psychosis for a longer time than in our study. Could it be that long DUP lead to more suicidal behaviour and thus covers the underlying gender difference? Studies finding more suicidal behaviour in females also report long DUPs, with medians ranging from 30 to 60 weeks (Bertelsen, Jeppesen et al. 2007; Cotton, Lambert et al. 2009; Barrett, Sundet et al. 2010). There are no differences in DUP between studies with and without gender differences in suicidal behaviour, and thus the length of DUP could not explain the gender differences in suicidal behaviour in FEP. This is in line with the conflicting results concerning the relationship between DUP and suicidal behaviour in FEP. Some studies report an association between longer DUP and suicide attempts (Altamura, Bassetti et al. 2003; Clarke, Whitty et al. 2006; Melle, Johannesen et al. 2006; Harvey, Dean et al. 2008; Barrett, Sundet et al. 2010), where other studies show no association between DUP and suicidal behaviour (Bakst, Rabinowitz et al. 2010; Nordentoft, Jeppesen et al. 2002; Foley, Jackson et al. 2008; Preti, Meneghelli et al. 2009). The median DUP of 16 weeks in our study reflects that the baseline data were collected at an early stage of the disorder. As shown in the TIPS study, early detection is clearly related to lower levels of suicidality (Melle, Johannesen et al. 2006).

The high levels of suicidality in females could also be seen in relation to their significantly higher scores on the depressive component of the PANSS, knowing that depression is a well documented risk factor for suicidal behaviour both in the general population and in psychotic disorders (Gupta, Black et al. 1998; Hawton, Sutton et al. 2005; Nock, Borges et al. 2008). In the logistic regression analysis both female gender and the depressive component of the PANSS retained significance. This makes female gender an independent risk factor for suicidal behaviour in this study, not only a result of the higher prevalence of depressive symptoms in females. 


\subsection{Gender as risk factor for suicidal behaviour}

A range of possible explanations for gender differences in suicidal behaviour in the general population have been put forward. Females tend to choose means of suicide less lethal than males i.e. poisoning, with a higher chance of surviving a suicide attempt (Beautrais 2002). The choice of suicide method does not necessarily reflect the suicidal intent. Similar methods may be used with very different intentions. The choice of suicide method is influenced by age, cultural values and the availability of means, as well as gender (Canetto and Sakinofsky 1998). Non-fatal suicidal behaviour in males may be associated with social stigma. The femininity attached to suicidal behaviour may lead to underreporting and masking of suicide attempts in males (Canetto 1997). Differences in recall and reporting of suicidal behaviour could also offer as an explanation for the gender differences. Females are better reporters of health history than males (Canetto and Sakinofsky 1998). Gender related differences in psychopathology could elucidate some of the gender differences in suicidal behaviour. Males have higher rates of externalizing disorders, like antisocial behaviour and substance abuse, while females dominate in internalizing disorders like depression and anxiety (Gasquet and Choquet 1993; Spirito, Bond et al. 1993; Brent 1995). The externalizing behaviours in males may contribute to more lethal, impulsive and determined suicide attempts with a higher mortality (Beautrais 2002). Even if females have higher rates of i.e. depression, the lower rates of suicide in females could be influenced by the gender pattern in help seeking behaviour. Females seek professional help to a larger extent than males (Moller-Leimkuhler 2002). These observations are from studies on the general population, and whether they can be generalized to FEP patients or not must be further explored.

Our regression analysis clearly showed that female gender is a significant risk factor for suicidality even when controlling for possible confounding variables. Thus, we believe that our study with a large, representative and consecutive sample of FEP patients show that the gender difference seen in the general population also is present in FEP patients. 


\subsection{Strengths and Limitations}

The main limitation of this study is the possibility of a recall bias in assessing suicidal behaviour retrospectively. We also had a rather high refusal rate $(40 \%)$ which could challenge the validity of our findings. The strength of this study is the participation of a large representative population based cohort of FEP patients with a fairly equal gender distribution.

\subsection{Conclusions}

In summary, we found high prevalence of suicidal behaviour in this sample of FEP. We also found a clear gender difference, with females reporting more suicidal behaviour both during lifetime and last month prior to study entry. This finding was significant even when controlling for change in premorbid social functioning, diagnosis, DUP, positive-, depressive- and cognitive PANSS components and drug abuse. 


\section{References}

Addington, J., J. Williams, et al. (2004). "Suicidal behaviour in early psychosis." Acta Psychiatrica Scandinavica 109(2): 116-20.

Altamura, A. C., R. Bassetti, et al. (2003). "Clinical variables related to suicide attempts in schizophrenic patients: a retrospective study." Schizophrenia Research 60(1): 47-55.

APA (2005). Diagnostic and Statistical Manual of Mental Disorders (DSM IV). Washington, DC, American Psychiatric Association.

Bakst, S., J. Rabinowitz, et al. (2010). "Antecedents and patterns of suicide behavior in first-admission psychosis." Schizophrenia Bulletin 36(4): 880-9.

Bakst, S., J. Rabinowitz, et al. (2010b). "Is poor premorbid functioning a risk factor for suicide attempts in first-admission psychosis?" Schizophrenia Research 116(2-3): 210-6.

Barrett, E. A., K. Sundet, et al. (2010). "Suicidality before and in the early phases of first episode psychosis." Schizophrenia Research 119(1-3): 11-17.

Beautrais, A. L. (2002). "Gender issues in youth suicidal behaviour." Emergency Medicine (Fremantle) 14(1): 35-42.

Beghi, M. and J. F. Rosenbaum (2010). "Risk factors for fatal and nonfatal repetition of suicide attempt: a critical appraisal." Current Opinion in Psychiatry 23(4): 349-55.

Bentsen, H., O. Munkvold, et al. (1996). "The interrater reliability of the positive and negative syndrome scale (PANSS)." International Journal of Methods in Psychiatric Research 6: 227-235.

Bernal, M., J. M. Haro, et al. (2007). "Risk factors for suicidality in Europe: results from the ESEMED study." Journal of Affective Disorders 101(1-3): 27-34.

Bertelsen, M., P. Jeppesen, et al. (2007). "Suicidal behaviour and mortality in firstepisode psychosis: the OPUS trial." The British Journal of Psychiatry Supplement 51: 140-6. 
Bertolote, J. M., A. Fleischmann, et al. (2005). "Suicide attempts, plans, and ideation in culturally diverse sites: the WHO SUPRE-MISS community survey." Psychological Medicine 35(10): 1457-65.

Brent, D. A. (1995). "Risk factors for adolescent suicide and suicidal behavior: mental and substance abuse disorders, family environmental factors, and life stress." Suicide and Life-Threatening Behavior 25 Supplement: 52-63.

Canetto, S. S. (1997). "Meanings of gender and suicidal behavior during adolescence." Suicide and Life-Threatening Behavior 27(4): 339-51.

Canetto, S. S. (2008). "Women and suicidal behavior: a cultural analysis." American Journal of Orthopsychiatry 78(2): 259-66.

Canetto, S. S. and I. Sakinofsky (1998). "The gender paradox in suicide." Suicide and Life-Threatening Behavior 28(1): 1-23.

Cannon-Spoor, H. E., S. G. Potkin, et al. (1982). "Measurement of premorbid adjustment in chronic schizophrenia." Schizophrenia Bulletin 8(3): 470-84.

Carlborg, A., J. Jokinen, et al. (2008). "Long-term suicide risk in schizophrenia spectrum psychoses: survival analysis by gender." Archives of Suicide Research 12(4): 347-51.

Carlborg, A., K. Winnerback, et al. (2010). "Suicide in schizophrenia." Expert Review of Neurotherapeutics 10(7): 1153-64.

Casey, P., G. Dunn, et al. (2008). "The prevalence of suicidal ideation in the general population: results from the Outcome of Depression International Network (ODIN) study." Social Psychiatry and Psychiatric Epidemiology 43(4): 299304.

Cavanagh, J. T., A. J. Carson, et al. (2003). "Psychological autopsy studies of suicide: a systematic review." Psychological Medicine 33(3): 395-405.

Clarke, M., P. Whitty, et al. (2006). "Suicidality in first episode psychosis." Schizophrenia Research 86(1-3): 221-5.

Cohen, S., J. Lavelle, et al. (1994). "Rates and correlates of suicide attempts in firstadmission psychotic patients." Acta Psychiatrica Scandinavica 90(3): 167-71. 
Cotton, S. M., M. Lambert, et al. (2009). "Gender differences in premorbid, entry, treatment, and outcome characteristics in a treated epidemiological sample of 661 patients with first episode psychosis." Schizophrenia Research 114(1-3): 17-24.

Cougnard, A., E. Kalmi, et al. (2004). "Pathways to care of first-admitted subjects with psychosis in South-Western France." Psychological Medicine 34(2): 26776.

Drake, R. E., F. C. Osher, et al. (1990). "Diagnosis of alcohol use disorders in schizophrenia." Schizophrenia Bulletin 16(1): 57-67.

Fenton, W. S. (2000). "Depression, suicide, and suicide prevention in schizophrenia." Suicide and Life-Threatening Behavior 30(1): 34-49.

First M, S. R., Gibbon M, Williams J. (1995). Structured Clinical Interview for DSMIV Axis I Disorders-Patient Edition (SCID I/P, Version 2.0). New York, New York State Psychiatric Institute, Biometrics Research Department

Foley, S., D. Jackson, et al. (2008). "Suicidality prior to presentation in first-episode psychosis." Early Intervention in Psychiatry 2(4): 242-6.

Gasquet, I. and M. Choquet (1993). "Gender role in adolescent suicidal behavior: observations and therapeutic implications." Acta Psychiatrica Scandinavica 87(1): 59-65.

Goldstein, J. M., S. L. Santangelo, et al. (1993). "Gender and mortality in schizophrenia: do women act like men?" Psychological Medicine 23(4): 941-8.

Gupta, S., D. W. Black, et al. (1998). "Factors associated with suicide attempts among patients with schizophrenia." Psychiatric Services 49(10): 1353-5.

Harris, E. C. and B. Barraclough (1997). "Suicide as an outcome for mental disorders. A meta-analysis." British Journal of Psychiatry 170: 205-28.

Harvey, S. B., K. Dean, et al. (2008). "Self-harm in first-episode psychosis." British Journal of Psychiatry 192(3): 178-84.

Haw, C., K. Hawton, et al. (2005). "Schizophrenia and deliberate self-harm: a systematic review of risk factors." Suicide and Life-Threatening Behavior 35(1): 50-62. 
Hawton, K., L. Sutton, et al. (2005). "Schizophrenia and suicide: systematic review of risk factors." British Journal of Psychiatry 187: 9-20.

Heila, H., E. T. Isometsa, et al. (1997). "Suicide and schizophrenia: a nationwide psychological autopsy study on age- and sex-specific clinical characteristics of 92 suicide victims with schizophrenia." American Journal of Psychiatry 154(9): 1235-42.

Hiroeh, U., L. Appleby, et al. (2001). "Death by homicide, suicide, and other unnatural causes in people with mental illness: a population-based study." Lancet 358(9299): 2110-12.

Hocaoglu, C. and Z. T. Babuc (2009). "Suicidal ideation in patients with schizophrenia." Israel Journal of Psychiatry and Related Sciences 46(3): 195203.

Inskip, H. M., E. C. Harris, et al. (1998). "Lifetime risk of suicide for affective disorder, alcoholism and schizophrenia." British Journal of Psychiatry 172: $35-$ 7.

Joa, I., J. O. Johannessen, et al. (2008). "The key to reducing duration of untreated first psychosis: information campaigns." Schizophrenia Bulletin 34(3): 466-72.

Joa, I., J. O. Johannessen, et al. (2009). "Baseline profiles of adolescent vs. adultonset first-episode psychosis in an early detection program." Acta Psychiatrica Scandinavica 119(6): 494-500.

Johannessen, J. O., I. Joa, et al. (2011). "First-episode psychosis patients recruited into treatment via early detection teams versus ordinary pathways: course and health service use during 5 years." Early Intervention in Psychiatry 5(1): 70-75.

Kay, S. R., A. Fiszbein, et al. (1987). "The positive and negative syndrome scale (PANSS) for schizophrenia." Schizophrenia Bulletin 13(2): 261-76.

Kerkhof, J. F. M. (2000). Attempted Suicide: Patterns and trends. In: The International Handbook of Suicide and Attempted Suicide. K. Hawton and K. Van Heeringen. Chichester, John Wiley \& Sons, Ltd.: 49-64. 
Kessler, R. C., G. Borges, et al. (1999). "Prevalence of and risk factors for lifetime suicide attempts in the National Comorbidity Survey." Archives of General Psychiatry 56(7): 617-26.

Koester, A., M. Lajer, et al. (2008). "Gender differences in first episode psychosis." Social Psychiatry and Psychiatric Epidemiology 43(12): 940-6.

Larsen, T. K., S. Friis, et al. (2004). "Premorbid adjustment in first-episode nonaffective psychosis: distinct patterns of pre-onset course." British Journal of Psychiatry 185: 108-15.

Larsen, T. K., T. H. McGlashan, et al. (1996). "First-episode schizophrenia: I. Early course parameters." Schizophrenia Bulletin 22(2): 241-56.

Larsen, T. K., I. Melle, et al. (2006). "Early detection of first-episode psychosis: the effect on 1-year outcome." Schizophrenia Bulletin 32(4): 758-64.

Larsen, T. K., I. Melle, et al. (2010). "Early detection of psychosis: positive effects on 5-year outcome." Psychological Medicine: 1-9.

Lester, D. (2006). "Sex differences in completed suicide by schizophrenic patients: a meta-analysis." Suicide and Life-Threatening Behavior 36(1): 50-6.

Melle, I., J. O. Johannesen, et al. (2006). "Early detection of the first episode of schizophrenia and suicidal behavior." American Journal of Psychiatry 163(5): 800-4.

Melle, I., J. O. Johannessen, et al. (2010). "Course and predictors of suicidality over the first two years of treatment in first-episode schizophrenia spectrum psychosis." Archives of Suicide Research 14(2): 158-70.

Melle, I., T. K. Larsen, et al. (2008). "Prevention of negative symptom psychopathologies in first-episode schizophrenia: two-year effects of reducing the duration of untreated psychosis." Archives of General Psychiatry 65(6): 634-40.

Melle, I., T. K. Larsen, et al. (2004). "Reducing the duration of untreated first-episode psychosis: effects on clinical presentation." Archives of General Psychiatry 61(2): 143-50. 
Moller-Leimkuhler, A. M. (2002). "Barriers to help-seeking by men: a review of sociocultural and clinical literature with particular reference to depression." Journal of Affective Disorders 71(1-3): 1-9.

Murray, C. J. and A. D. Lopez (1996). "Evidence-based health policy--lessons from the Global Burden of Disease Study." Science 274(5288): 740-3.

Nock, M. K., G. Borges, et al. (2008). "Cross-national prevalence and risk factors for suicidal ideation, plans and attempts." British Journal of Psychiatry 192(2): 98105.

Nordentoft, M., P. Jeppesen, et al. (2002). "OPUS study: suicidal behaviour, suicidal ideation and hopelessness among patients with first-episode psychosis. Oneyear follow-up of a randomised controlled trial." British Journal of Psychiatry Supplement 43: 98-106.

Palmer, B. A., V. S. Pankratz, et al. (2005). "The lifetime risk of suicide in schizophrenia: a reexamination." Archives of General Psychiatry 62(3): 24753.

Pompili, M., X. F. Amador, et al. (2007). "Suicide risk in schizophrenia: learning from the past to change the future." Annals of General Psychiatry 6: 22.

Preston, N. J., K. G. Orr, et al. (2002). "Gender differences in premorbid adjustment of patients with first episode psychosis." Schizophrenia Research 55(3): 28590.

Preti, A., A. Meneghelli, et al. (2009). "Risk of suicide and suicidal ideation in psychosis: results from an Italian multi-modal pilot program on early intervention in psychosis." Schizophrenia Research 113(2-3): 145-50.

Radomsky, E. D., G. L. Haas, et al. (1999). "Suicidal behavior in patients with schizophrenia and other psychotic disorders." American Journal of Psychiatry 156(10): 1590-5.

Reutfors, J., L. Brandt, et al. (2009). "Risk factors for suicide in schizophrenia: findings from a Swedish population-based case-control study." Schizophrenia Research 108(1-3): 231-7. 
Robinson, J., S. Cotton, et al. (2009). "Prevalence and predictors of suicide attempt in an incidence cohort of 661 young people with first-episode psychosis." The Australian and New Zealand Journal of Psychiatry 43(2): 149-57.

Robinson, J., M. G. Harris, et al. (2010). "Suicide attempt in first-episode psychosis: a 7.4 year follow-up study." Schizophrenia Research 116(1): 1-8.

Spirito, A., A. Bond, et al. (1993). "Gender differences among adolescent suicide attempters." Crisis 14(4): 178-84.

Tarrier, N., C. Barrowclough, et al. (2004). "Risk of non-fatal suicide ideation and behaviour in recent onset schizophrenia--the influence of clinical, social, selfesteem and demographic factors." Social Psychiatry and Psychiatric Epidemiology 39(11): 927-37.

Thorup, A., L. Petersen, et al. (2007). "Gender differences in young adults with firstepisode schizophrenia spectrum disorders at baseline in the Danish OPUS study." Journal of Nervous and Mental Disease 195(5): 396-405.

Tidemalm, D., N. Langstrom, et al. (2008). "Risk of suicide after suicide attempt according to coexisting psychiatric disorder: Swedish cohort study with long term follow-up." BMJ 337: 6.

Weissman, M. M., R. C. Bland, et al. (1999). "Prevalence of suicide ideation and suicide attempts in nine countries." Psychological Medicine 29(1): 9-17.

Welch, S. S. (2001). "A review of the literature on the epidemiology of parasuicide in the general population." Psychiatric Services 52(3): 368-75.

WHO (2004). The global burden of disease: 2004 update. Geneva, World helath organization: $1-146$.

WHO. (2011). "Mental health." Suicide rates 04/07/2011. Retrieved 04/07/2011, 2011, from http://www.who.int/mental_health/prevention/suicide_rates/en/index.html.

WHO. (2011b). "Suicide prevention." Mental health Retrieved 04/07/2011, 2011, from

http://www.who.int/mental_health/prevention/suicide/suicideprevent/en/index. html. 
